\title{
Extending the Functionality of On-line PD Monitoring Equipment for MV Power Cables
}

\author{
Y. $\mathrm{Li}^{1}$, P.A.A.F. Wouters ${ }^{1}, \mathrm{P}$. Wagenaars ${ }^{2}$ \\ 1) Eindhoven University of Technology, the Netherlands \\ 2) DNV GL, Arnhem, the Netherlands
}

\begin{abstract}
An on-line partial discharge (PD) monitoring and location system for medium voltage cable circuits was developed previously. This paper explores ways to extend its range of application. The extension includes PD location method by time domain reflectometry (TDR) when reflections are not easily identifiable. The functionality of the PD monitoring equipment can also be widened by sensing other quantities related to the condition of the cable insulation using the same device. Dynamic cable temperature monitoring can be achieved by recording variation in the high frequency signal propagation velocity along the cable. Water ingress in paper-insulated lead-covered (PILC) cable decreases the cable's characteristic impedance while it increases the permittivity of the insulation. It can be observed by changes in the reflection pattern from the cable or by a lowered propagation velocity. Instead of recording reflection patterns in time domain, a frequency domain approach based on an impedance scan is investigated to be applied for cables in service.
\end{abstract}

\section{Introduction}

The on-line partial discharge (PD) monitoring and location system for medium voltage cable circuits described in [1, 2] consists of two pulse injection units integrated with sensors at both cable ends. The PD location is derived from the difference in time-of-arrival of the PD pulse at each end of the cable. Synchronisation of the sensor recordings is achieved by injecting reference pulses at both ends which serve as time stamps in the measured patterns.

This paper aims to extend the range of application, both in options to locate PDs and by adding functionality besides PD diagnostics. To this end, the presence of the pulse injection units at both cable sides is exploited. It allows for having reference patterns to be compared with signals caused by PD events.

Injected pulses can act as fingerprints to locate PD signals based on TDR when reflections in the patterns have not a known origin. This situation occurs when unexpected reflections arise from changes in cable impedance (e.g. different cable types). Also expected reflection from an cable end may be absent if the configuration is such that the cable behaves as being more or less characteristically terminated. This can happen for cables connected to the rest of the grid.
Different approaches are discussed in Section 2 and checked with experiments in a small $10 \mathrm{kV}$ network. Dynamic cable temperature monitoring can help the utilities in asset management and maintenance. The high frequency signal propagation velocity along the cable is affected by the cable temperature. This feature can be exploited to monitor the dynamic cable temperature. Section 3 discusses both laboratory tests and field data. Water ingress is a main reason for the failure of PILC cable. Water decreases the cable's characteristic impedance, which changes the reflection pattern from the cable. If the water enters a considerable cable length, it also can be observed in the propagation time of the injected signal. These aspects are investigated in Section 4 both experimentally and through simulation. The perspectives of frequency domain diagnostic approach instead of time domain measurement are investigated. It is based on an impedance scan made at the terminals of an injection coil, which is magnetically coupled to the cable under test. Impedance discontinuities can be revealed, indicating possible defects. Section 5 discusses results of laboratory experiments to explore the feasibility of such approach.

\section{Single-sided PD monitoring}

Single-sided detection could occasionally be preferred above double-sided measurements. Besides the obvious reduction of needed equipment, also practical issues as available space to install sensors in a ring-main-unit (RMU) can urge to opt for a single-sided solution. Two approaches are proposed to realize a single-sided solution for a cable connection in service. One method is based on an extra sensor at one side to enable directional sensing for arrival of pulses. The other method relies on additional ferrite material to provide a clear recognizable reference reflection pulse.

The integrated injection unit sends pulses on a regular basis, e.g. from RMU1 as indicated in Figure 1a. The sensor at the same end as the injection unit will detect reflected pulses arising from impedance variations along the cable. These reflections, together with the injected pulse itself, form a pattern: the "reference pattern". Similarly, a "PD pattern" arises at the same sensor if a PD occurs somewhere along the cable. As the impedance variation points are physically fixed, the reflection pulses from these points will have unique features both in the "PD pattern" and the "reference pattern". By comparing these patterns, it is possible to locate the PD origin. The principle is shown in Figures 
$1 \mathrm{~b}$ and 1c. They show the direct reflections together with the main secondary reflections which are indicated with a superscript (2). The signals from beyond joint $\mathrm{x}$ and $\mathrm{z}$ are omitted to simplify the discussion.

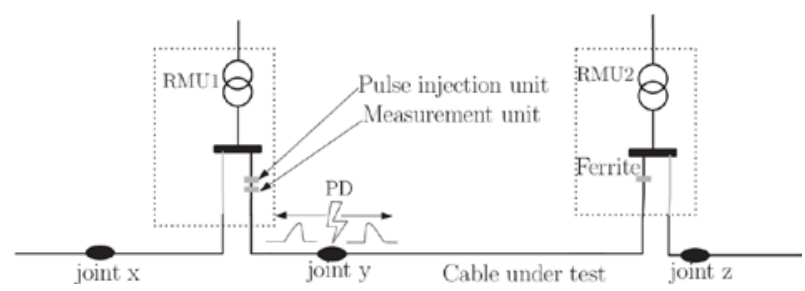

(a) Scheme of the method of single-sided detection.

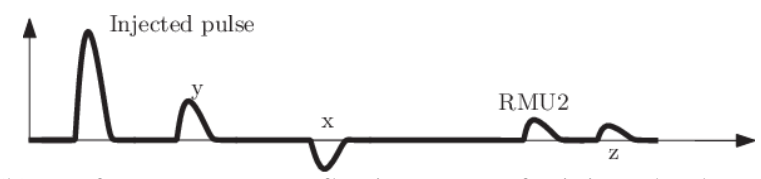

(b) Reference pattern: reflection pattern for injected pulse.

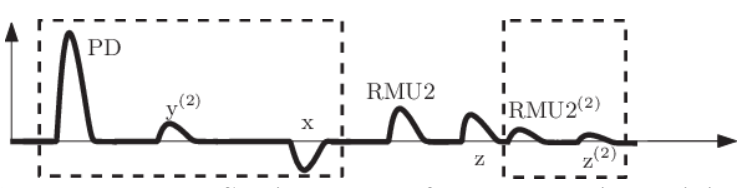

(c) PD pattern: reflection pattern for PD occurring at joint y (aligned with injected pulse pattern). Signal inside dashed block part contains corresponding locations to the reference pattern.

Fig. 1 - Principle of partial discharge location with single sensor; signals are detected at RMU1.

Figure $1 \mathrm{~b}$ shows the reference pattern recorded by the measurement unit upon the injected pulse from the pulse injection unit. The signal polarity here only serves to simplify the explanation. Figure 1c gives the "PD pattern”, originating from a PD occurring at joint $y$. Two approaches are proposed utilizing the features in the "reference pattern" and "PD pattern”.

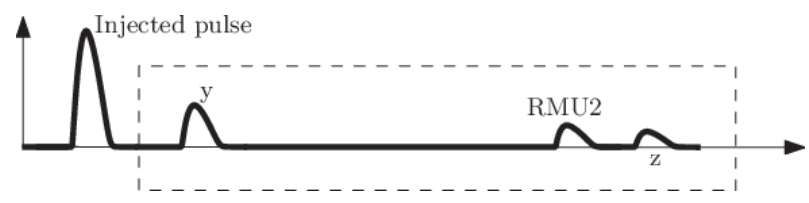

(a) Reference pattern after directional sensing (reflections from left side are removed). Signal in dashed block is used for cross correlation.

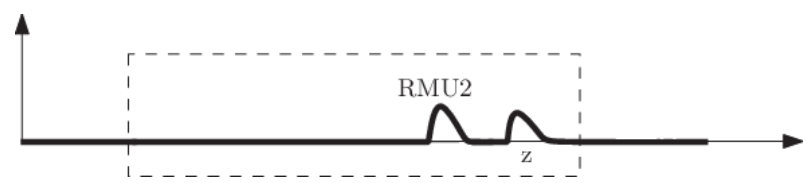

(b) $\mathrm{PD}$ pattern after removal of reflections which have the same location as reference pattern in Figure 1b. Signal in dashed block is used for cross correlation.

Fig. 2 - Principle of PD location with single sensor; signals are detected at RMU1.
The first approach to locate the PD is to get rid of all reflections in Figure 1c that are also included in Figure $1 \mathrm{~b}$ at the same time of arrival. The part that will be eliminated is indicated in Figure 1c inside the dashed block. Next, the "reference pattern" in Figure 1b is processed with directional filtering by specifically designed transfer functions [3,4]. Figure 2a shows the "reference pattern" after directional filtering. Compared to Figure $1 \mathrm{~b}$ it lacks the reflection from joint $\mathrm{x}$. This elimination optimizes the "reference pattern" for comparison with the "PD pattern". From the "PD pattern" shown in Figure 2b, the peaks which also appear in Figure 1b are eliminated. The resulting "PD pattern" contains only features regarding the PD location. The PD defect can be located by cross correlation. The shift needed for the "PD pattern" with respect to the "reference pattern" to maximize the cross correlation provides the PD location.

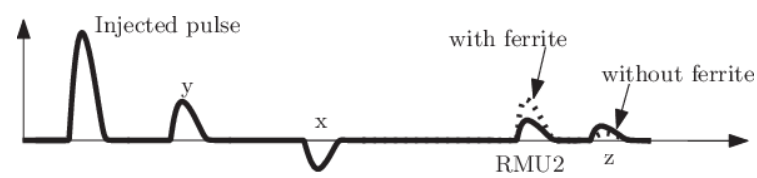

Fig. 3 - Conceptual illustration for ferrite effect to the reference reflection pattern.

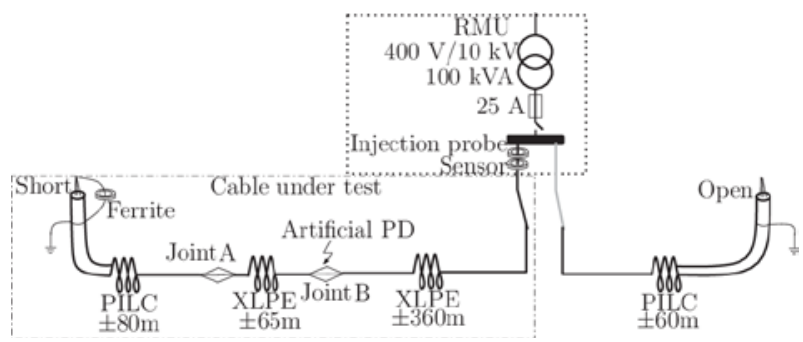

Fig. 4 - Test circuit for single-sided PD location.

For the second approach a reference position is created to facilitate the pattern correlation between the "reference pattern" and "PD pattern". This can be accomplished by clamping a ferrite core at the other end of the cable under test. It enhances the far end reflection because ferrite material adds extra impedance. The ferrite provides a reference point in the "reflection pattern" and in the "PD pattern", making the alignment of both patterns easier. The ferrite effect is illustrated in Figure 3 for the "reflection pattern" in Figure 1b. The ferrite acts also for the "PD pattern". Reference [4] discusses a practical implementation of "switching" the ferrite impedance. It is based on a winding around the ferrite which can be switched between open and closed loop. In open state the ferrite adds impedance to the detection circuit. In closed state the magnetic field induced by the loop current compensates the magnetic flux in the ferrite, reducing its added impedance. To recognize the ferrite location in the $\mathrm{PD}$ pattern, at least two PDs have to be recorded: with and without added ferrite impedance. 


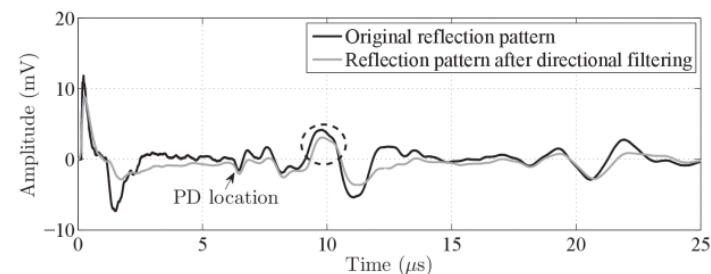

(a) Directional filtering applied to the "reference pattern".

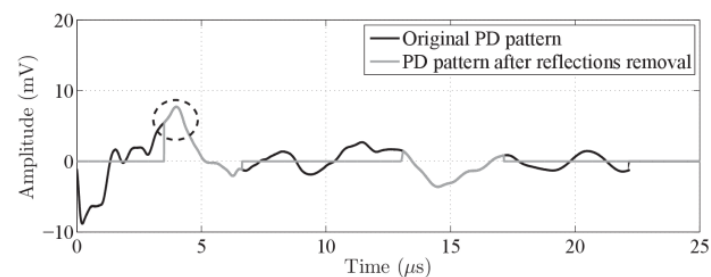

(b) Reflection removal applied to the "PD pattern".

Fig. 5 - Reference reflection pattern and PD pattern prepared for cross correlation with directional filtering.

Measurements are conducted in the full scale test set-up representing a typical Dutch RMU with MV cables as depicted in Figure 4. The cable from the left far end to the RMU is taken as the cable under test. Figure 5 shows the result for the directional sensing method. Note that the negative reflection from the right open end immediately after the injection pulse around $1.5 \mu \mathrm{s}$ is eliminated from the pattern, as shown in Figure 5a. The "PD pattern" is obtained from the artificial PD source (pulse via signal generator) in Figure 4. From this pattern the reflections with the same locations as in the "reference pattern" are removed. The patterns indicated in grey in Figure 5 are used for cross correlation. The matching points between the two patterns are indicated with dotted circles in the patterns. Figure 6 shows the experiment result for the approach based on adding ferrite material. It can be seen that for the "reference pattern", the ferrite location appears around $10.0 \mu \mathrm{s}$ while for "PD pattern", it locates around $4.0 \mu \mathrm{s}$. With this reference reflection point, the "reference pattern" and "PD pattern" can be aligned to locate the PD. Experiments show that both approaches can locate the PD origin with uncertainty in the range of $2 \%$ with respect to the travelling time from $\mathrm{PD}$ source to sensor.

\section{Temperature monitoring}

Dynamic thermal rating can help utilities for planning and operation of power systems. For high voltage underground power cable, optic fibres can be implemented in the cable screen or in a pipe laid close to the centre phase [5]. However, the large number of distributed MV cables makes this solution unaffordable on the distribution level.

For MV cables equipped with defect location based on PD activity, information on high frequency signal propagation along the power cable is acquired [1]. Variability in propagation velocity can in principle be employed to detect temperature variation due to e.g. cable loading. This solution can be combined with PD monitoring, extending the PD monitoring functionality to include temperature sensing at relatively low investment costs.

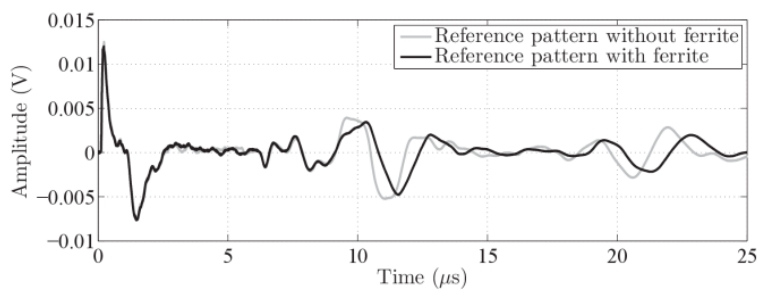

(a) Ferrite material affects "reference pattern".

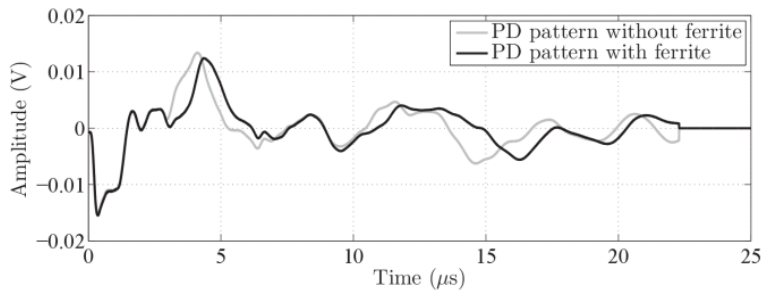

(b) Ferrite material affects "PD pattern".

Fig. 6 - Reference pattern and PD pattern with and without ferrite at cable far end.

The effect of temperature on both XLPE and oilimpregnated paper insulation is investigated. Connections with both cable insulation types were heated and the propagation velocities were measured as function of temperature. Test results show that high frequency signals propagate faster with higher temperatures for XLPE and slower for PILC cable. To investigate the cause for the temperature dependent velocity, the permittivity of samples from both insulation materials is measured at different temperatures [6]. The permittivity is extracted from the sample impedance measured by a Network Analyzer. Based on the relation:

$$
v \cong \frac{1}{\sqrt{\varepsilon_{0} \varepsilon_{r} \mu_{0}}}
$$

the permittivity is converted to velocity. The result is compared with the measured temperature dependent velocity as shown in Figure 7.

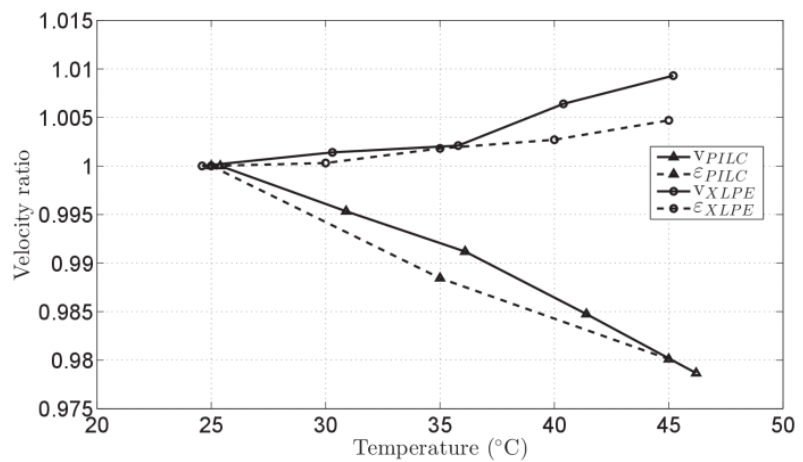

Fig. 7 - Comparison of relative change in velocity with temperature, based on the velocity measurements and the permittivity measurements for both XLPE and PILC cable; the starting temperature is around $25^{\circ} \mathrm{C}$. Note that these temperatures are derived for the insulation midpoint. 
As an example the propagation time for a specific PILC cable section with $1464 \mathrm{~m}$ length is extracted from the monitoring system and the load current is derived from substation recordings. Figure 8 shows the week cycle comparison between the propagation time for the cable section and the load recorded at the substation both in summer and in winter. It can be seen that the propagation time profile follows the load profile. Higher temperature results in lower propagation velocity, which is consistent with laboratory measurement. The variation of propagation time in Figure 7 is approximately linear and a typical $0.2 \%$ variation in propagation time corresponds to $5^{\circ} \mathrm{C}$ variation in temperature both during summer and winter. Reference [6] shows an example for XLPE cable. There the reverse relationship between propagation velocity and temperature is confirmed.

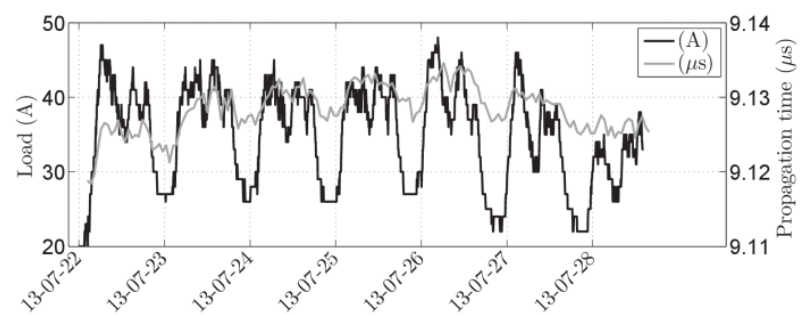

(a) Propagation time and load of PILC cable for week cycle in summer.

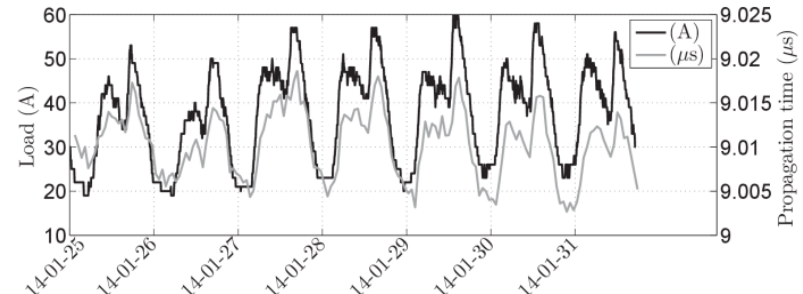

(b) Propagation time and load of PILC cable for week cycle in winter.

Fig. 8 - Propagation time and load of PILC cable for week cycle. Maximum current rating for this cable circuit is around 190 A.

\section{Water ingress monitoring}

Moisture is one of the most common causes of deterioration to the properties of oil-impregnated paper insulation [7]. This paper focuses on on-line water ingress detection for long PILC cable section. The water ingress process is studied in a laboratory scale setup.

A segment of $80 \mathrm{~m}$ MV PILC cable is used for the study. It consists of three circular conductors embedded in oil-paper insulation surrounded by a lead earth sheath. At one end the TDR measurements are performed, whereas at the other end water ingress takes place. Beyond the water ingress point there remains about $20 \mathrm{~m}$ of cable. A hole is made in the lead earth screen to expose the oil-impregnated paper to moisture. A compressor forces water at 1.5 bar into the paper insulation. Karl Fischer titration technique is commonly used to monitor the moisture of the paper insulation, but it needs to extract a paper sample which would destroy the cable segment [8]. Instead, a commercial humidity sensor (SHT71 sensor for air humidity) is applied. The sensor measures the relative humidity. Four humidity sensors with $1 \mathrm{~m}$ distance from each other are installed in the oil-impregnated paper insulation.

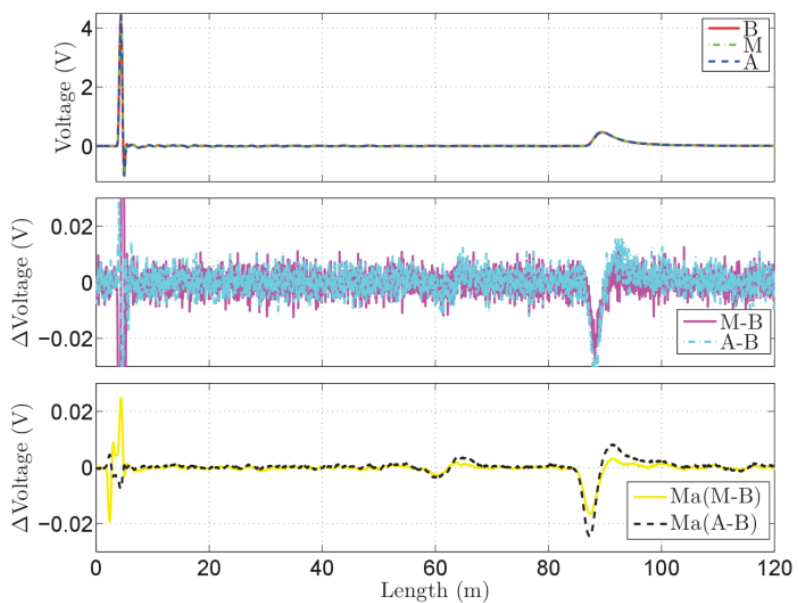

Fig. 9 - TDR measurement before (B), midway (M) and after (A) the water ingress experiment; $\mathrm{M}-\mathrm{B}$ is the difference between pattern $\mathrm{M}$ and $\mathrm{B}, \mathrm{A}-\mathrm{B}$ is the difference between pattern $A$ and $B ; M a(M-B)$ means the moving average filter is applied to the result of $\mathrm{M}-\mathrm{B}, \mathrm{Ma}(\mathrm{A}-\mathrm{B})$ means moving average filter is applied to the result of A-B.

For the TDR measurements, a pulse width of $8 \mathrm{~ns}$ is applied. Comparison between the three recorded patterns is shown in Figure 9. Though in the original TDR patterns no direct observable change can be found, the difference between two patterns shows a peak at the water injection point (around $60 \mathrm{~m}$ ). To further reduce noise, average filtering is applied to the differential patterns. A window with length corresponding to $2 \mathrm{~m}$ cable length is shifted along the result in Figure 9. A running average value within the window length is taken. The negative peak in the bottom figure around $60 \mathrm{~m}$ indicates that water decreases the cable impedance. The permittivity for a mixture, according to [9] is given by

$$
\varepsilon_{r w}=\varepsilon_{\mathrm{H}_{2} 0}+2 \cdot c \cdot \varepsilon_{\mathrm{H}_{2} 0} \frac{\varepsilon_{r}-\varepsilon_{\mathrm{H}_{2} 0}}{\varepsilon_{r}+\varepsilon_{\mathrm{H}_{2} 0}-c \cdot\left(\varepsilon_{r}-\varepsilon_{\mathrm{H}_{2} 0}\right)},
$$

where $\varepsilon_{r w}$ is the relative permittivity of the mixture: $\varepsilon_{r}$ (3.5) for the dry oil-impregnated paper, $\varepsilon_{H 2 O}$ (78.5) for the relative permittivity of water, and $c$ is the volume concentration of the oil-impregnated paper. Fraction 1-c is the volume concentration of the water.

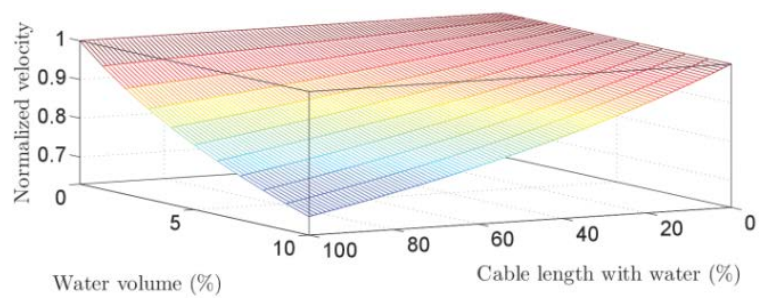

Fig. 10 - Relative velocity as function of water volume concentration and percentage of cable with water ingress. 
In case water enters the cable over a considerable length, it has a measurable effect. Figure 10 shows the average normalized velocity as a function of the amount of water ingress over a percentage of the cable length.

\section{Frequency domain cable diagnostics}

Inductive coupling is employed to allow for on-line impedance measurement. A bulk current injection probe (BCIP) can be installed to obtain strong mutual coupling with circuits inside an RMU. The impedance observed at the BCIP terminals, the primary circuit, depends essentially on the impedance from the circuit around which this coil is placed, the secondary circuit. This allows for observation of any changes in RMU and connected cables, which causes a change of impedance. To extract this information from the observed BCIP impedance a calibration procedure is required. The BCIP can be modelled as a "T" structure with primary side impedance $Z_{1}$, secondary side impedance $Z_{2}$ and mutual impedance $Z_{m}$, as depicted in Figure 11 . Calibration is achieved by placing the BCIP in a calibration fixture. From the input impedance of the fixture measured by loading the BCIP with open, short and $50 \Omega$ the three impedances are obtained [10].

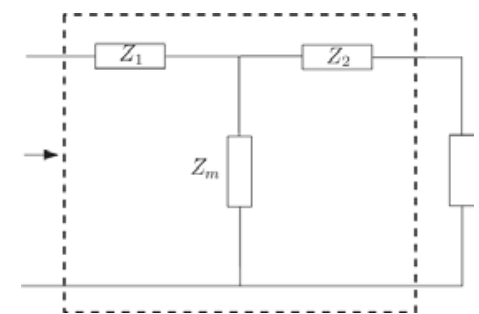

Fig. 11 - Model for bulk current injection probe (BCIP).

When the BCIP is clamped around a circuit, its input impedance will be determined by the circuit load. The frequency dependent circuit load can then be extracted from the measured input impedance of BCIP. Afterwards, the frequency domain load impedance can be converted to an equivalent TDR result via the inverse Fourier transform. Any impedance variation changes the TDR result starting as from that specific location. Thus the impedance variation location can be identified.

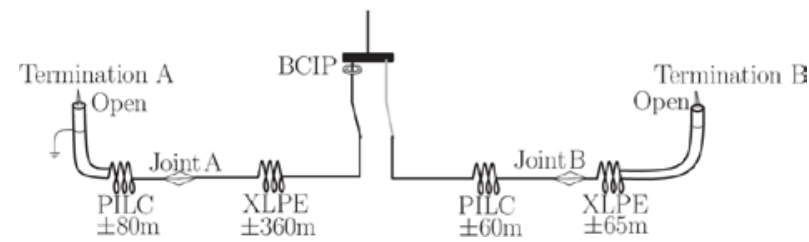

Fig. 12 - Test circuit for frequency domain impedance scan.

The detectability of local variations of the impedance along a cable connection is investigated. It is based on a frequency scan of the cable impedance observed at the cable end inside an RMU. The test circuit shown in Figure 12 consists of an RMU with two connected cables. There are cable joint positions at $360 \mathrm{~m}$ on the left (transition XLPE to PILC cable) and at $60 \mathrm{~m}$ on the right (PILC to XLPE). At the far ends or at these joints, impedance variations can be introduced. The BCIP is situated at the busbar as shown in Figure 12. Its input impedance is measured with the Network Analyzer and subsequently converted to time domain. By employing an average propagation velocity for the combined XLPE and PILC connection of $120 \mathrm{~m} / \mathrm{s}$, the waveforms shown in Figure 13 are plotted as functions of distance. The arrows point to positions which correspond to the joints $\mathrm{A}$ and $\mathrm{B}$.
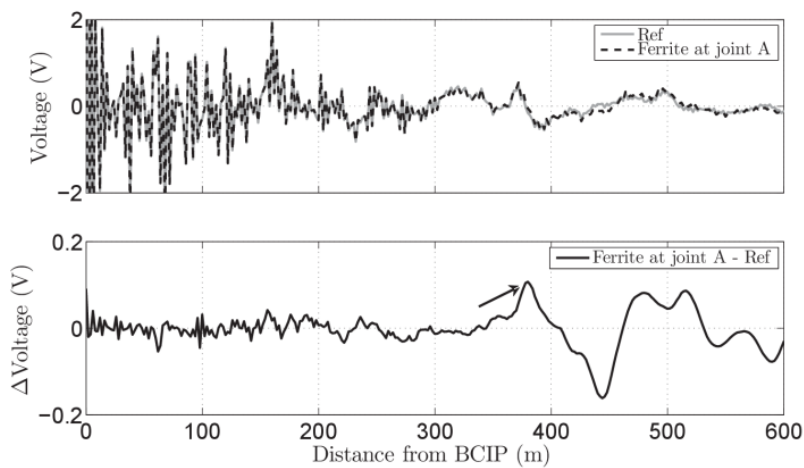

(a) Reference pattern and pattern with ferrite around joint $\mathrm{A}$.
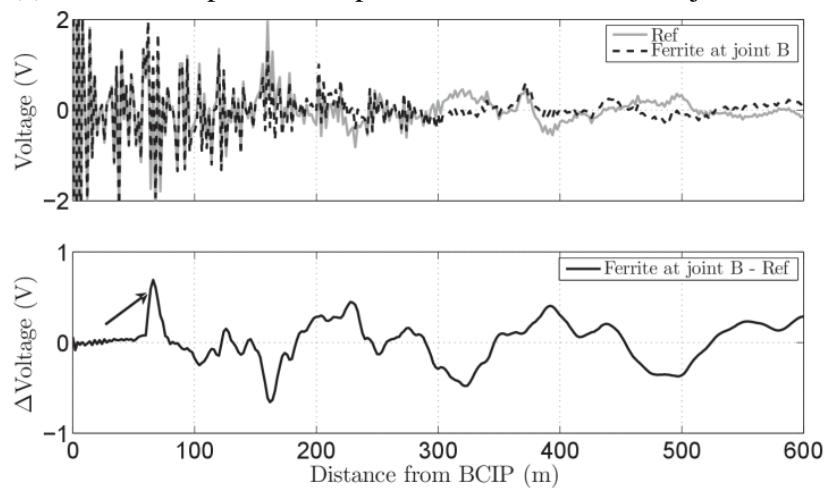

(b) Reference pattern and pattern with ferrite around joint $\mathrm{B}$.

Fig. 13 - Frequency domain impedance scan for cable impedance variation at joints; (a) the inverse Fourier transform from the impedance scan for reference measurement and specific impedance variation, (b) the differences between them.

\section{Conclusions}

An approach to realize single-sided PD monitoring for cables in service is proposed in the paper. The method can simplify the system and widen the application scenarios. Two approaches are proposed. Both of them are based on comparing a reference pattern obtained from an injected signal to patterns from a PD. The first approach relies on transfer functions for achieving directional sensing. The second approach is based on added impedance by placing ferrite material at a defined place in the connection to provide a reference reflection point. Experiments show that both approaches can locate the PD origin successfully with uncertainty in the order of $2 \%$. 
High frequency signals propagate faster with higher temperatures for XLPE cable and slower for PILC. It is observed that the temperature effect on propagation velocity is more significant for PILC (around 1\% velocity change for $10^{\circ} \mathrm{C}$ variation) than for XLPE (around $0.5 \%$ velocity change for $10^{\circ} \mathrm{C}$ variation). Measurements indicate non-linearity in the temperature dependence of the propagation velocity in XLPE insulated cables. This effect can be exploited to get realtime recording of the cable insulation temperature without the need of additional investment.

The effect of water ingress to PILC cable impedance is studied experimentally. Moisture in the insulation material decreases the characteristic impedance, leading to a pulse reflection variation. The magnitude of the reflection is a measure for water penetration. Significant reflection occurs at a low percentage of volume concentration. It seems feasible to observe concentrations of water ingress below critical level. Important factors determining the sensitivity are the length over which ingress has taken place and the distance with respect to the detection side. Alternatively, if water ingress has taken place over a considerable fraction of the total connection length (e.g. $10 \%$ ), it may be observed through a change of the overall signal propagation time.

A different diagnostic method, based on impedance scan is proposed in this paper. The method can be implemented for on-line application by scanning the input impedance of a BCIP around the earth connection. A change of impedance in the cable will be reflected in the inverse Fourier transform of the measured frequency spectrum of the cable impedance. The proposed approach is validated with a small scale MV network and the impedance variation is successfully detected over a distance up to about $400 \mathrm{~m}$.

\section{References}

[1] P.C.J.M. van der Wielen, J. Veen, P.A.A.F. Wouters, and E.F. Steennis, "Online partial discharge detection of $\mathrm{mv}$ cables with defect localisation (pdol) based on two time synchronised sensors", 18th International Conference and Exhibition on Electricity Distribution (CIRED 2005), pp. 1-5, June 2005.
[2] P.C.J.M. van der Wielen and E.F. Steennis, "Experiences with diagnostics on complete inservice mv cable feeders”, Electrical Insulation Conference (EIC), pp. 349-353, June 2011.

[3] P. Wagenaars, P.C.J.M. van der Wielen, P.A.A.F. Wouters and E.F. Steennis, "Directional Sensing for Online PD Monitoring of MV Cables", Nordic Insulation Symposium, pp. 79-82, Trondheim, Norway, June 2005.

[4] Y. Li, P.A.A.F. Wouters, P. Wagenaars, P.C.J.M. van der Wielen, E.F. Steennis, "Single-Sided Partial Discharge Location Method Based on Impedance Discontinuities along Power Cable", 18th Power Systems Computation Conference, Wroclaw, Poland, August 2014.

[5] R. Olsen, J. Holboell, and U.S. Gudmundsdottir, "Electrothermal coordination in cable based transmission grids”,. IEEE Transactions on Power Systems, 28(4):4867 - 4874, 2013.

[6] Y. Li, P.A.A.F. Wouters, P. Wagenaars, P.C.J.M. van der Wielen, E. F. Steennis, “Temperature Dependency of Wave Propagation Velocity in MV Power Cable”, 18th International Symposium on High Voltage Engineering, Seoul, August 2013.

[7] R. Neimanis and R. Eriksson, "Diagnosis of moisture in oil/paper distribution cables - part i: Estimation of moisture content using frequencydomain spectroscopy”, IEEE Transactions on Power Delivery, 19(1):9 - 14, Jan 2004.

[8] M. Wang, S.M. Rowland, and P.E. Clements, "Moisture ingress into low voltage oil-impregnated paper insulated distribution cables”, IET Science, Measurement Technology, 1(5):276 - 283, Sept 2007.

[9] K.K. Karkkainen, A.H. Sihvola, and K.I. Nikoskinen, "Effective permittivity of mixtures: numerical validation by the fdtd method", IEEE Transactions on Geoscience and Remote Sensing, 38(3):1303 - 1308, May 2000.

[10] Z.L. Dong, Y. Li, P.A.A.F. Wouters, P. Wagenaars, E.F. Steennis, "Underground power cable diagnostics: online defect positioning”, accepted for IEEE PowerTech 2015, Eindhoven, June-July, 2015. 